15.2;06.4

\title{
Прямые измерения динамики электрокалорического отклика сегнетоэлектриков в условиях произвольного теплообмена
}

\author{
(С Г.Ю. Сотникова, Г.А. Гаврилов, А.А. Капралов, Е.П. Смирнова \\ Физико-технический институт им. А.Ф. Иофрфе РАН, Санкт-Петербург, Россия \\ E-mail: g.sotnikova@mail.ioffe.ru
}

Поступило в Редакцию 7 июня 2019 г.

В окончательной редакции 11 июня 2019 г.

Принято к публикации 17 июня 2019 г.

\begin{abstract}
Предложен новый эффективный метод прямых измерений электрокалорического эффекта при произвольных условиях теплообмена. Точность и достоверность измерений динамики изменения температуры образца обусловлены использованием фотодиодных температурных сенсоров среднего ИК-диапазона спектра с возможностью их in situ калибровки.
\end{abstract}

Ключевые слова: электрокалорический эффект, ИК-сенсоры температуры, сегнетоэлектрики-релаксоры.

DOI: 10.21883/PJTF.2019.19.48308.17912

Последние годы отмечены растущим интересом к изучению электрокалорического эффекта (ЕСЕ) в сегнетоэлектриках. ЕСЕ является обратным пироэлектрическому эффекту и рассматривается как новое решение для разработки эффективных накопителей энергии и охлаждающих устройств [1-4]. Изучение прямых и обратных эффектов преобразования тепловой энергии в электрическую в полярных материалах представляет также фундаментальный интерес, поскольку является инструментом для анализа природы поляризационных процессов. Количественными характеристиками, определяющими способность материала к преобразованию тепловой энергии, являются пироэлектрический коэффициент $p$ и электрокалорическое изменение температуры $\delta T$ при заданном электрическом поле $E$. Повышение метрологической достоверности измерений этих параметров всегда было и остается актуальной экспериментальной задачей. Разработан ряд аналитических методов изучения пиро- и электрокалорического эффектов в материалах различных типов: от объемных монокристаллов и керамики [5] до тонких пленок [2]. Все известные методы можно разделить на прямые и косвенные с точки зрения процедуры получения данных о динамике изменения температуры образца $[1,6]$. Как показывает анализ публикаций по ЕСЕ, результаты исследований, выполненных различными методами, имеют значительный разброс. Так, например, значения $\delta T$, полученные для керамики $\mathrm{Ba}_{0.65} \mathrm{Sr}_{0.35} \mathrm{TiO}_{3}$ в калориметрах разных типов и с помощью ИК-камеры [7], различаются почти в 3 раза. Значительные расхождения в величине $\delta T$ для одного и того же материала указывают на недостаточную достоверность результатов, подавляющее большинство которых получено с помощью косвенных методов (до $85 \%$ [1]) или температурных датчиков с недостаточными динамическими свойствами [8]. Наиболее достоверными методами измерений ЕСЕ в настоящее время считаются калориметрические методы, в которых реализуются условия измерений, близкие к адиабатическим. Однако, используемые при этом вычислительные модели для расчета $\delta T$ не учитывают теплообмен образца с внешней средой, что приводит к дополнительным погрешностям [6,8]. Таким образом, и в случае калориметрических измерений прямые методы измерения температуры предпочтительны [8], а в условиях произвольного теплообмена они являются единственно возможными. Теплообмен выступает в качестве обязательного условия практического применения исследуемых материалов, так как для эффективного извлечения тепла рабочий элемент преобразователя (накопителя энергии/охладителя) одновременно должен обеспечивать возможность приложения достаточно сильного электрического поля и обладать достаточной массой. В случае теплового контакта большой площади значение коэффициента теплопередачи $H$, характеризующее эффективность теплообмена между образцом и окружающей средой, может достигать величины $3400 \mathrm{~W} \cdot \mathrm{m}^{-2} \cdot \mathrm{K}^{-1}$ [9], в то время как для условий свободной конвекции воздуха типичные значения $H \approx 25-30 \mathrm{~W} \cdot \mathrm{m}^{-2} \cdot \mathrm{K}^{-1}$ [10]. Сегодня прямые методы измерения температуры в ЕСЕ-исследованиях осуществляются в основном контактными датчиками (типа pt-100) или коммерчески доступными ИК-камерами. При этом вопросам надежности и достоверности этих измерений уделяется незаслуженно мало внимания. В настоящей работе предлагается новый метод прямых бесконтактных измерений температуры образца с использованием радиационных сенсоров среднего $(2-5 \mu \mathrm{m})$ ИК-диапазона (MIR-сенсоры) на основе фотодиодов (ФД) $\mathrm{A}^{3} \mathrm{~B}^{5}$ в качестве чувствительного элемента. Высокая спектральная чувствительность и селективность $\left(\Delta \lambda / \lambda_{m} \sim 0.1\right)$, обусловленные использованием flip-chip-конструкции ФД и иммерсионной оптики [11], позволяют использовать для описания их работы в качестве датчика температуры монохроматическую модель на основе закона Планка. Это дает очевидную 


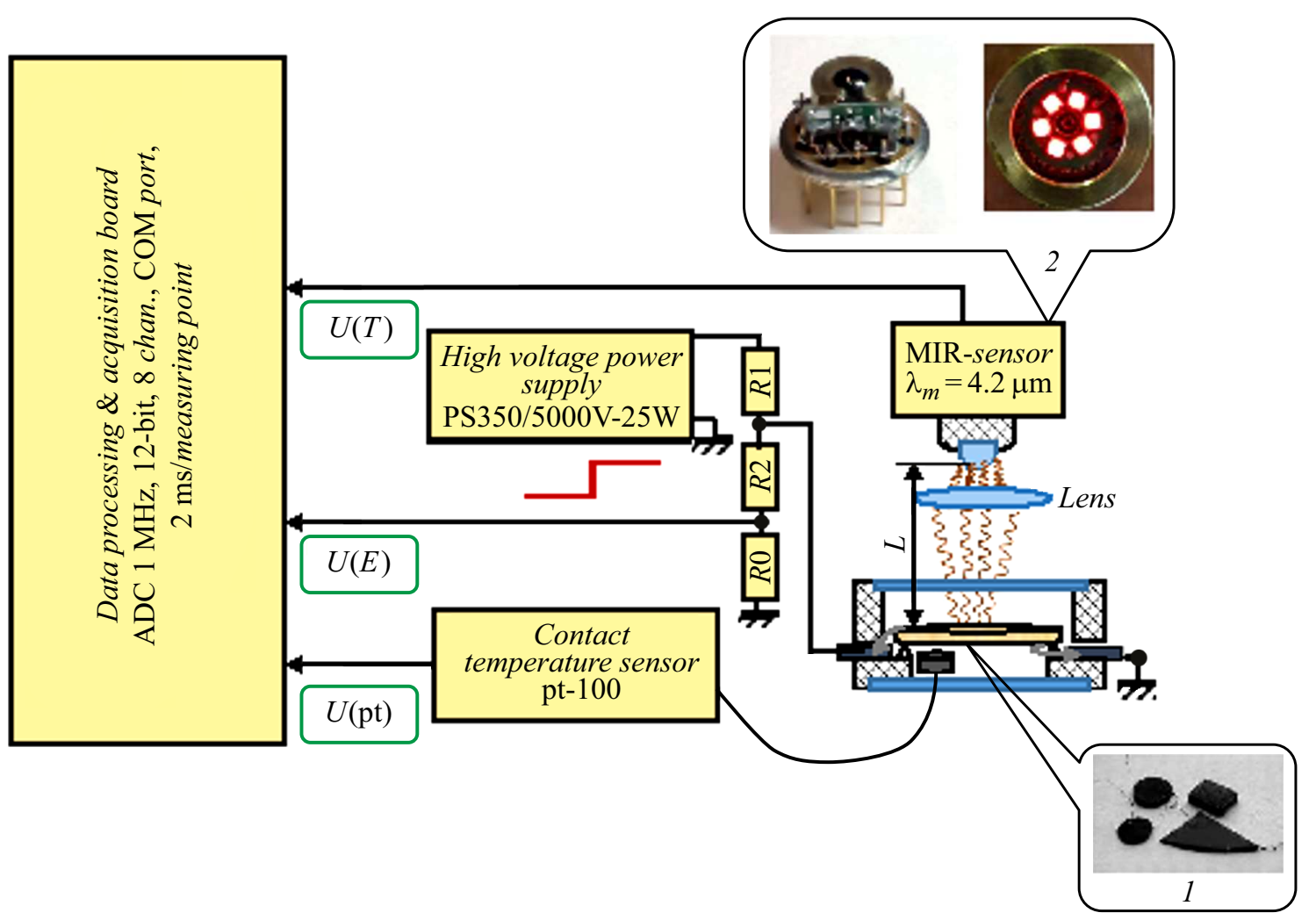

Pис. 1. Схема эксперимента для прямых измерений электрокалорического отклика с использованием MIR-сенсора.

функциональную связь фототока ФД $\left(I_{p h}\right)$ с температурой поверхности исследуемого объекта $(T)$, которая в приближении Вина для $\lambda T<3000 \mu \mathrm{m} \cdot \mathrm{K}$ имеет вид

$$
\begin{aligned}
I_{p h}(T) & =B S\left(\lambda_{e f f}\right) K\left(\lambda_{e f f}\right) \frac{C 1}{\lambda_{e f f}^{5}} \\
& \times \exp \left(-\frac{C 2}{\lambda_{e f f} T}\right)=\theta \exp \left(-\frac{C 2}{\lambda_{e f f} T}\right),
\end{aligned}
$$

где $C 1$ и $C 2$ - постоянные Планка, $B$ - площадь поверхности объекта, на которой измеряется температура, $S\left(\lambda_{e f f}\right)$ - токовая фоточувствительность ФД, $K\left(\lambda_{e f f}\right)-$ коэффициент передачи теплового излучения, зависящий от пропускания промежуточной среды и излучательной способности объекта, $\lambda_{e f f}$ - эффективная длина волны измерений, зависящая от ширины спектральной характеристики ФД и диапазона измеряемых температур.

Именно неопределенность параметра $\theta$ является основным источником погрешности измерения температуры коммерчески доступными ИК-сенсорами, так как условия градуировки, выполняемой производителем, не могут точно соответствовать условиям реального эксперимента. С другой стороны, линейность зависимости фототока от интенсивности детектируемого излучения и модель MIR-сенсора в виде (1) позволяют выполнять in situ калибровку (уточнение параметра $\theta$ ) перед началом каждого эксперимента по одному устойчивому температурному состоянию образца, контролируемому эталонным (контактным) датчиком. Это устраняет систематические ошибки, характерные при использовании известных ИК-сенсоров, ограничивая погрешность измерений только шумами детектора MIR-сенсора. Дополнительным преимуществом MIR-сенсоров является их нечувствительность к засветкам с $\lambda<1 \mu \mathrm{m}$, что позволяет контролировать температуру объекта непосредственно в области воздействия на него лазерного излучения. Это было использовано нами ранее в лазерных тепловых методиках для определения коэффициента теплоотдачи $H$ объемных материалов [10].

Схема эксперимента для прямых измерений температуры образца в ЕСЕ-исследованиях представлена на рис. 1. В качестве детектирующего элемента MIR-сенсора использовался иммерсионный ФД типа $\mathrm{PD} 42 \mathrm{Sr}$ (ООО „ИоффеЛЕД“, www.ioffeled.com), чувствительный в узкой спектральной области $\lambda_{m} \pm \Delta \lambda=4.1 \pm 0.2 \mu \mathrm{m}$. Дополнительная сапфировая линза обеспечивала измерение температуры на участке поверхности диаметром $\sim 5 \mathrm{~mm}$ на расстоянии $L=30 \mathrm{~m}$, что давало возможность контроля температуры образцов, в том числе в камерах калориметров и термостатов. Экспериментальные значения неопределенности показаний MIR-сенсора при быстродействии $1 \mathrm{~ms}$ составили $50 \mathrm{mK}$ при измерениях около $20^{\circ} \mathrm{C}$, менее $5 \mathrm{mK}$ в области $100^{\circ} \mathrm{C}$ и менее $1 \mathrm{mK}$ при $200^{\circ} \mathrm{C}$ и более, что было ограничено только шумами элементной базы операционных усилителей и требуемым быстродействием [12]. В наших экспериментах об- 

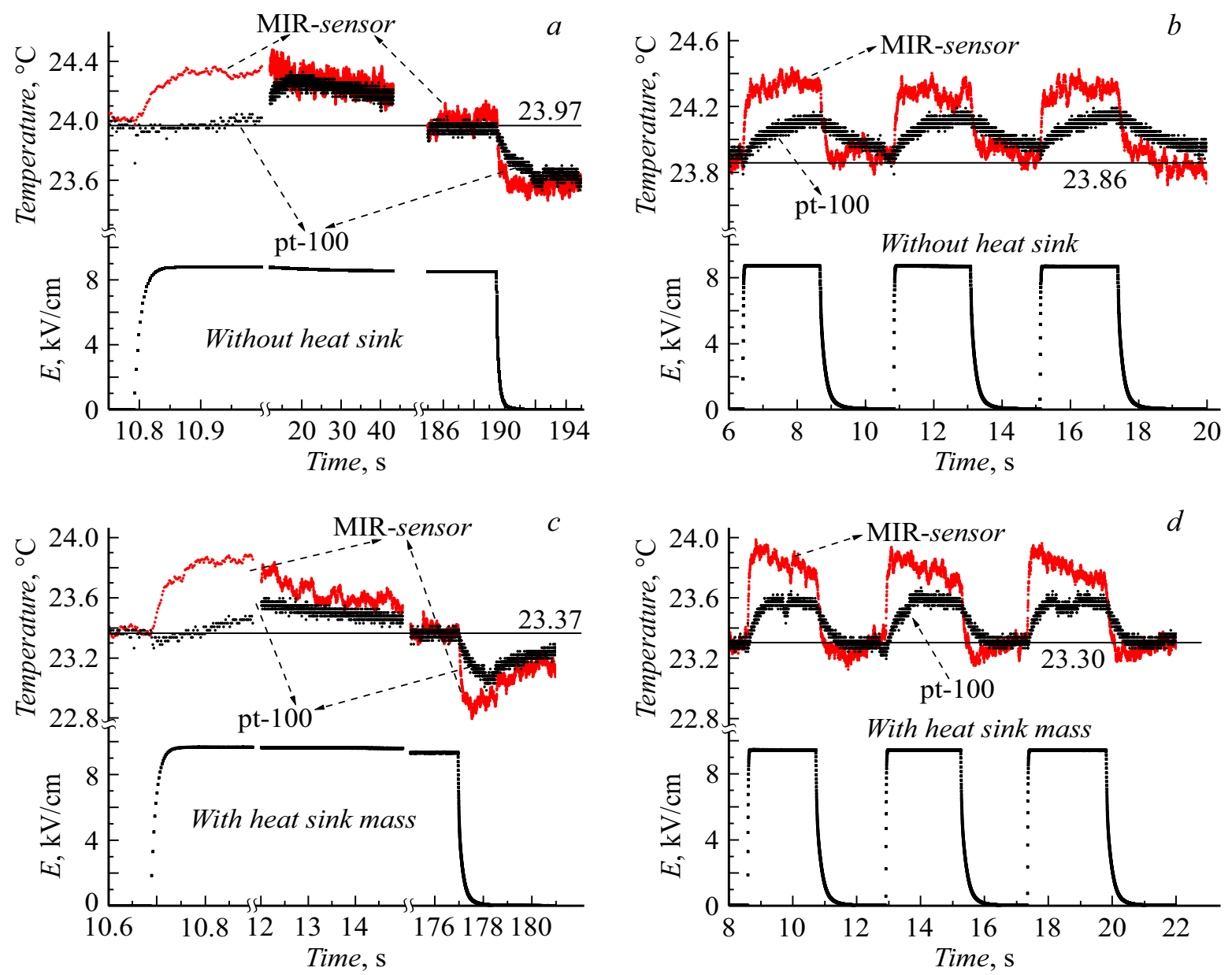

Рис. 2. Электрокалорические отклики свободного образца $(a, b)$ и образца, одна из поверхностей которого находится в тепловом контакте со стальной пластинкой размером $10.6 \times 8.7 \times 2 \mathrm{~mm}(c, d)$, при различных формах электрического воздействия $E(t)$.

разцы различной толщины и формы (вставка 1 на рис. 1$)$ помещались в кювету с сапфировыми окнами, где свободно лежали на кольцевом держателе. На противоположные поверхности образца наносились электроды для подачи электрического поля и слой поглощающего материала для увеличения его излучательной способности. Электронное обрамление MIR-сенсора, смонтированное в едином с ФД корпусе ТО-8 с термохолодильником (вставка 2 на рис. 1), обеспечивало измерение фототока ФД и термостабилизацию параметров ФД и первого усилительного каскада. Встроенный светодиодный визир, установленный в плоскости иммерсионной линзы ФД, позволял контролировать размер и положение области измерения на поверхности образца. Контактный температурный датчик pt-100 (HERAEUS, www.heraeus.com) использовался для in situ калибровки MIR-сенсора и для сравнения его измерительных возможностей с предлагаемым MIR-сенсором. Цифровая часть схемы позволяла осуществлять одновременное аналого-цифровое преобразование и передачу данных от используемых в эксперименте сенсоров с частотой выборок $1 \mathrm{kHz}$. Для иллюстрации возможностей метода в работе приведены результаты измерения динамики ЕСЕ-отклика образца керамического материала (сегнетоэлектрика-релаксора PMN-PSN) размером $7.6 \times 9.8 \times 1.2 \mathrm{~mm}$. На рис. 2 представлены ЕСЕ-отклики образца, находящегося в кювете в условиях свободного конвективного теплообмена (рис. 2, $a, b$ ), и при установке образца на теплоотвод (рис. $2, c, d$ ) в виде стальной пластины размером $10.6 \times 8.7 \times 2 \mathrm{~mm}$ при приложении/снятии электрического поля. Из рис. 2, $а$ видно, что при использовании MIR-сенсора в условиях конвективного теплообмена при подаче на образец импульса электрического поля $E=9.5 \mathrm{kV} / \mathrm{cm}$ температура на его поверхности резко возрастает, $\delta T_{\text {on }}=0.50 \pm 0.05 \mathrm{~K}$, а затем медленно падает до исходной температуры с постоянной времени $\tau=2 H / \rho C L \approx 50 \mathrm{~s}(\rho-$ плотность, $C-$ теплоемкость, $L$ - толщина образца), соответствующей коэффициенту $H=27 \mathrm{~W} \cdot \mathrm{m}^{-2} \cdot \mathrm{K}^{-1}$ ), измеренному экспериментально для данного образца по методике, описанной в [10]. При снятии электрического поля наблюдается обратный скачок температуры: $\delta T_{\text {off }}=\delta T_{\text {on }}$. При наличии дополнительного теплоотвода (рис. 2, $c$ и $d$ ) также наблюдаются резкие скачки температуры $\delta T_{o n}=\delta T_{\text {off }}$ 
при значительно более быстрых временах релаксации к температуре окружающей среды. В обоих случаях амплитуда $\delta T$ хорошо совпадает со значением, полученным для образца аналогичного материала в квазиадиабатическом калориметре [13]. Из рис. 2 видно, что быстродействия контактного сенсора pt-100 явно недостаточно для контроля быстрых изменений температуры, характерных для процессов, ответственных за ЕСЕ. Более того, инерционность pt-100 зависит от условий теплоотвода (www.heraeus.com), поэтому она не только влияет на измеряемую амплитуду $\delta T$, но и существенно искажает истинную картину динамики ЕСЕ на образце в условиях различного теплоотвода.

Приведенные результаты убедительно доказывают, что предлагаемый новый метод исследования динамики электрокалорического отклика, основанный на прямых измерениях температуры образца бесконтактным быстродействующим MIR-сенсором, открывает широкие возможности для изучения природы ECE, обеспечивает необходимую точность и достоверность измерений и позволяет оценить перспективность материалов для практических приложений в элементах преобразователей тепловой энергии, работающих в существенно неадиабатических условиях.

\section{Финансирование работы}

Работа поддержана Российским фондом фундаментальных исследований (грант 18-02-00394).

\section{Конфликт интересов}

Авторы заявляют, что у них нет конфликта интересов.

\section{Список литературы}

[1] Liu Y., Scott J.F., Dkhil B. // Appl. Phys. Rev. 2016. V. 3. N 3. P. 031102 (1-19). DOI: 10.1063/1.4958327

[2] Pandya S., Wilbur J., Kim J., Gao R., Dasgupta A., Dames C., Martin L.W. // Nature Mater. 2018. V. 17. N 5. P. 432-438. DOI: $10.1038 / \mathrm{s} 41563-018-0059-8$

[3] Zhang T., Qian X.-S., Gu H., Hou Y., Zhang Q.M. //Appl. Phys. Lett. 2017. V. 110. N 24. P. 243503 (1-4). DOI: $10.1063 / 1.4986508$

[4] Electrocaloric materials: new generation of cooler / Eds T. Correia, Q. Zhang. Springer, 2013. 253 p.

DOI: 10.1007/978-3-642-40264-7

[5] Plaznik U., Kitanovski A., Rožič B., Malič B., Uršič H., Drnovšek S., Cilenšek J., Vrabelj M., Poredoš A., Kutnjak Z. // Appl. Phys. Lett. 2015. V. 106. N 4. P. 043903 (1-4). DOI: $10.1063 / 1.4907258$

[6] Sanlialp M., Shvartsman V.V., Faye R., Karabasov M.O., Molin C., Gebhardt S., Defay E., Lupascu D.C. // Rev. Sci. Instrum. 2018. V. 89. N 3. P. 034903 (1-7). DOI: $10.1063 / 1.4997155$

[7] Rokosz M.K. // Metrology of the electrocaloric effect based on an infrared imaging technique. PhD Thesis. London: Imperial College, 2016. $191 \mathrm{p}$.

spiral.imperial.ac.uk/handle/10044/1/47999
[8] Бондарев В.С., Михалева Е.А., Флеров И.Н., Горев М.В. // ФTT. 2017. T. 59. B. 6. C. 1097-1105. DOI: 10.21883/PJTF.2019.19.48308.17912

[9] Faye R., Strozyk H., Dkhil B., Defay E. // J. Phys. D: Appl. Phys. 2017. V. 50. N 46. P. 464002 (1-7). DOI: $10.1088 / 1361-6463 /$ aaa7c4/meta

[10] Александров С.Е., Гаврилов Г.А., Капралов А.А., Муратиков К.Л., Сотникова Г.Ю. // Письма в ЖТФ. 2017. Т. 43. B. 14. C. $104-110$. DOI: 10.21883/PJTF.2019.19.48308.17912

[11] Brunkov P.N., Ilinskaya N.D., Karandashev S.A., Lavrov A.A., Matveev B.A., Remennyi M.A., Stus' N.M., Usikova A.A. // Infrared Phys. Technol. 2015. V. 73. P. 232-237. DOI: $10.1016 /$ j.infrared.2015.09.017

[12] Aleksandrov S.E., Gavrilov G.A., Kapralov A.A., Sotnikova G.Y. // 4th Int. Conf. of photonics and information optics. Phys. Procedia. 2015. V. 73. P. 177-182.

[13] Смирнова Е.П., Сотникова Г.Ю., Зайцева Н.В., Капралов А.А., Гаврилов Г.А., Сотников А.В. // ФТТ. 2018. Т. 60. B. 10. C. 1964-1968. DOI: 10.21883/FTT.2018.10.46524.111 